\title{
Spin precession due to spin-orbit coupling in a two-dimensional electron gas with spin injection via ideal quantum point contact
}

\author{
Ming-Hao Liu, Ching-Ray Chang, and Son-Hsien Chen \\ Department of Physics, National Taiwan University, Taipei 106, Taiwan \\ (Received 30 July 2004; revised manuscript received 20 January 2005; published 12 April 2005)
}

\begin{abstract}
We present the analytical result of the expectation value of spin with respect to an arbitrarily spin-polarized electron state which is injected via an ideal quantum point contact into a semi-infinite two-dimensional electron gas confined in a [001]-grown quantum well. Both the Rashba and Dresselhaus spin-orbit couplings are taken into account. The spatial behaviors of the spin precession due to the Rashba term, the Dresselhaus term, and simultaneously both terms are analyzed. We demonstrate that the [110], [110], [1 $\overline{1} 0]$, and $[1 \overline{1} 0]$ axes own invariant behavior of spin precession, which is the same as that due to the Rashba term.
\end{abstract}

DOI: 10.1103/PhysRevB.71.153305

PACS number(s): 72.25.Dc, 71.70.Ej, 85.75.Hh

Recent research on spin-polarized electron transport in semiconductors has attracted great attention in the emerging field of spintronics. ${ }^{1}$ Of particular interest is the manipulation of spin via spin-orbit (SO) coupling in semiconductor nanostructures. In a two-dimensional electron gas (2DEG) confined in a heterostructured quantum well (QW), two basic mechanisms of the SO coupling have been often taken into account: (i) the structure inversion asymmetry (SIA) mechanism described by the Rashba term, ${ }^{2}$

$$
H_{R}=\frac{\alpha}{\hbar}\left(p_{x} \sigma_{y}-p_{y} \sigma_{x}\right)
$$

whose coupling strength $\alpha$ is gate-voltage dependent, ${ }^{3-9}$ and (ii) the bulk inversion asymmetry (BIA) mechanism described by the Dresselhaus term. ${ }^{10,11}$ When restricted to a two-dimensional semiconductor nanostructure with [001] growth geometry, this term is of the form, ${ }^{12,13}$

$$
H_{D}=\frac{\beta}{\hbar}\left(p_{x} \sigma_{x}-p_{y} \sigma_{y}\right),
$$

where the coupling parameter $\beta$ is material specific. The interface inversion asymmetry ${ }^{14,15}$ (IIA) also provides a certain contribution to the Dresselhaus term in the SO coupling, but it is phenomenologically inseparable from BIA.

Whereas the competition between the Rashba and Dresselhaus terms was concluded with the result that the former dominates in narrow-gap systems, ${ }^{3,4}$ while the latter dominates in wide-gap materials, ${ }^{16}$ Datta and Das proposed the theoretical idea of constructing an electronic analog of the optic modulator using ferromagnetic contacts as the spin injector and the detector, with a 2DEG channel confined in a narrow-gap semiconductor, where only the Rashba SO coupling taken into account. ${ }^{17}$ In their proposal, the spin precession was envisioned due to the interference between the two eigenfunctions, superposing the wave function of the injected spin with a gate-voltage-tunable phase difference $\Delta \theta$ $=2 m^{*} \alpha L / \hbar^{2}$, with $L$ being the channel length. Therefore, the spin-orientation angle for electrons arriving at the end of the 2DEG channel, and hence the resulting current, is theoretically tunable via the applied gate voltage. Hence the pro- posed device was expected to serve as a field-effect transistor (FET) based on the electron spin and has been commonly referred to as the Datta-Das spin-FET.

Recently, Winkler further demonstrated this well known spin precession described above and also the spin orientation in a quasi-two-dimensional (quasi-2D) electron system by using an $8 \times 8$ Kane model, which takes into account both the SIA and the BIA mechanisms. ${ }^{18}$ The spin orientation was shown to be sensitively dependent on the crystallographic direction in which the quasi-2D system is grown. This was also consistent with the previous results obtained by Lusakowski et al. showing that the conductance of the Datta-Das spin-FET depends significantly on the crystallographic direction of the channel when the Dresselhaus term is also present. ${ }^{19}$ Indeed, the contribution to the SO coupling of the Rashba and the Dresselhaus terms may be of the same order in some QWs [such as GaAs QWs (Ref. 20)] and their ratio was even shown to be experimentally determinable very recently. ${ }^{21}$ Therefore, the possible effects caused by the Dresselhaus term on spin-related devices has been an imperative issue in semiconductor spintronics. ${ }^{19,22}$

In this paper, we extend Winkler's work, ${ }^{18}$ in which he calculated the expectation value of the spin operator $\langle\mathbf{S}\rangle$ with respect to the injected spin-polarized electron state. Whereas the spin precession was shown by calculating the overlaps between the spin vector and the polarization of the ferromagnetic drain contact numerically, we present the analytical result of the spin vector as a function of the coupling strengths $\alpha$ and $\beta$, the orientation angle of the injected spin, and the position of determination. A pictorial interpretation of the spin precession along certain transport directions is given. By analyzing the two extreme cases, pure Rashba and pure Dresselhaus, the spin precession due to SO couplings in inversion-asymmetric 2DEGs can be understood more concretely. Some crystallographic directions with interesting and handleable spin-precession behavior are found and may imply certain applicability in spintronics.

Consider an electron with a definite spin perfectly injected via an ideal point contact [set on the origin $(0,0)]$ into an inversion-asymmetric 2DEG, where both the Rashba and Dresselhaus SO couplings are present. The 2DEG is assumed to be semi-infinite so that the boundary effect is out of consideration. Let the electron be injected at an angle $\phi$ with 
spin $\mathbf{S}_{0}$ orienting toward $\phi_{s}$, with respect to the $x$ axis. Setting the growth direction of the 2DEG layer to be [001], and the $x$ and $y$ axes to be [100] and [010], respectively, the single electron Hamiltonian for $x>0$ (within which the semiinfinite 2DEG is set up) under the effective mass approximation can be written as $H=\left(p^{2} / 2 m^{*}\right) \sigma_{0}+H_{R}+H_{D}$ where $m^{*}$ is the electron effective mass in the 2DEG, and $\sigma_{0}$ is the 2 $\times 2$ unit matrix. Note that $H_{R}$ and $H_{D}$ given by Eqs. (1) and (2), which are preferably described by a multiband approach, are used. These two common expressions are in fact the lowest-order terms when the multiband equations are transformed to one-band equations. ${ }^{18}$ Defining

$$
\gamma(\phi) \equiv \sqrt{\alpha^{2}+\beta^{2}+2 \alpha \beta \sin 2 \phi}, \quad e^{-i \varphi} \equiv \frac{\alpha e^{-i \phi}-i \beta e^{i \phi}}{\gamma(\phi)},
$$

the corresponding eigenenergies and eigenfunctions can be easily obtained as

$$
\begin{gathered}
E_{ \pm}=\frac{\left(\hbar k_{\|}^{ \pm}\right)^{2}}{2 m^{*}} \pm \gamma(\phi) k_{\|}^{ \pm}, \\
\left\langle\mathbf{r} \mid \mathbf{k}_{\|}^{ \pm}, \pm\right\rangle \equiv \frac{1}{\sqrt{2}} e^{i \mathbf{k}_{\|}^{ \pm} \cdot \mathbf{r}}\left(\begin{array}{c}
i e^{-i \varphi} \\
\pm 1
\end{array}\right),
\end{gathered}
$$

where the in-plane wave vector $\mathbf{k}_{\|}$and the position vector $\mathbf{r}$ represent the two-dimensional vectors $\left(k_{x}, k_{y}\right)$ and $(x, y)$, respectively. Note that the eigenenergies and eigenstates are written in a way slightly different from the previously obtained ones ${ }^{18,23}$ for convenience, which will be clearer later. Separating the spin part from the state kets $\left|\mathbf{k}_{\|}^{ \pm}, \pm\right\rangle$, we denote the eigenspinors as $|\varphi-\pi / 2, \pm\rangle$ with the usual definition $|\widetilde{\alpha}, \pm\rangle \equiv|\widetilde{\beta}=0, \widetilde{\alpha}, \pm\rangle=\left(e^{i \widetilde{\alpha}} \pm 1\right)^{\dagger} / \sqrt{2}$, where $\widetilde{\beta}$ and $\widetilde{\alpha}$ are the polar and azimuthal angles, respectively. ${ }^{24}$ Taking Eq. (4b) as the basis, the injected spin $\left|\phi_{s},+\right\rangle$ can be expanded as $\left|\phi_{S},+\right\rangle=c_{+}|\varphi-\pi / 2,+\rangle+c_{-}|\varphi-\pi / 2,-\rangle, \quad$ where $\quad c_{ \pm}=\langle\varphi$ $-\pi / 2, \pm\left|\phi_{s},+\right\rangle=\left(-i e^{i\left(\varphi-\phi_{s}\right)} \pm 1\right) / 2$. Since there is a phase difference, $\Delta \theta(\mathbf{r})=2 m^{*} \gamma(\phi) r / \hbar^{2}$ between $|\varphi-\pi / 2,+\rangle$ and $\mid \varphi$ $-\pi / 2,-\rangle$, the spin state ket at position $\mathbf{r}$ can be equivalently written as $\left|\phi_{s},+\right\rangle_{\mathbf{r}}^{R D}=c_{+} e^{-i(\Delta \theta(\mathbf{r}) / 2)}|\varphi-\pi / 2,+\rangle+c_{-} e^{i(\Delta \theta(\mathbf{r}) / 2)} \mid \varphi$ $-\pi / 2,-\rangle$ where the superscript "RD" denotes that both the Rashba and Dresselhaus terms are nonvanishing. By computing the expectation values of $\mathbf{S}$ with respect to the state ket $\left|\phi_{s},+\right\rangle_{\mathbf{r}}^{R D}$, regardless of the factor $\hbar / 2$, we obtain

$$
\begin{aligned}
\langle\mathbf{S}\rangle_{\mathbf{r}}^{R D} & \equiv\left(\begin{array}{l}
\left\langle S_{x}\right\rangle_{\mathbf{r}}^{R D} \\
\left\langle S_{y}\right\rangle_{\mathbf{r}}^{R D}
\end{array}\right) \\
& =\left(\begin{array}{l}
\cos \phi_{s} \cos ^{2} \frac{\Delta \theta}{2}-\cos \left(2 \varphi-\phi_{s}\right) \sin ^{2} \frac{\Delta \theta}{2} \\
\sin \phi_{s} \cos ^{2} \frac{\Delta \theta}{2}-\sin \left(2 \varphi-\phi_{s}\right) \sin ^{2} \frac{\Delta \theta}{2}
\end{array}\right) .
\end{aligned}
$$

Note that the phase difference $\Delta \theta$ is, in general, dependent of $\phi$ in the presence of both the Rashba and the Dresselhaus terms, and is isotropic only when either $\alpha$ or $\beta$ vanishes, as will be seen in the later discussion. In the following we dis- (a)

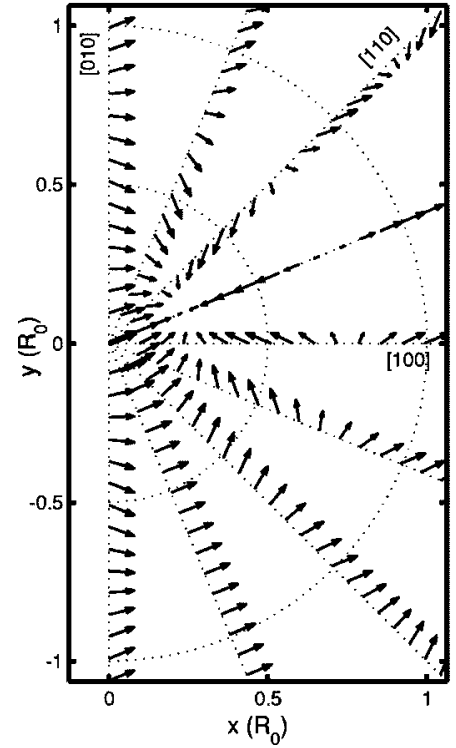

(b)

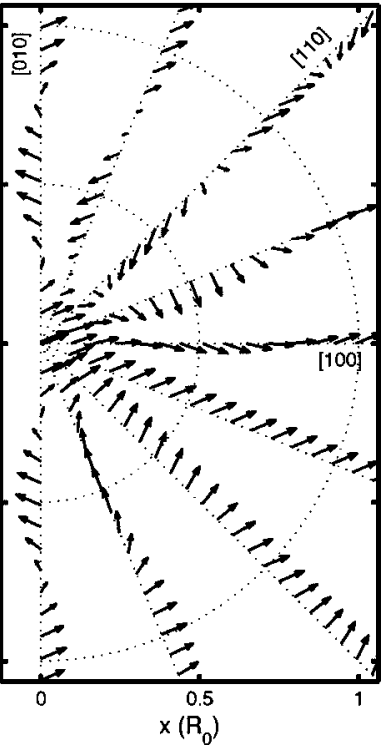

FIG. 1. Spin precession due to (a) Rashba and (b) Dresselhaus spin-orbit couplings in a 2DEG. Each arrow indicates $\langle\mathbf{S}\rangle_{\mathbf{r}}$ on the corresponding space point. The injected spin, shown by the bold arrow on $(0,0)$, is set to orient $\pi / 8$ with respect to [100]. The compact unit $R_{0}$ is defined in Eq. (6), and the dotted lines are guides for the eyes.

cuss the behavior of the spin precession under two extreme cases: $(\alpha \neq 0, \beta=0)$ and $(\alpha=0, \beta \neq 0)$.

In the absence of the Dresselhaus term, we return to the familiar results of the Rashba case, $\gamma(\beta=0)=\alpha$. Here the reason we set Eq. (3) is clear since the mathematical forms of the eigenvalues, eigenfunctions, and the expectation values of $\mathbf{S}$ expressed in Eqs. (4) and (5) are maintained. The results for this case are obtained simply by replacing $\varphi$ with $\phi$, e.g., the expectation value of $\mathbf{S}$ is $\langle\mathbf{S}\rangle_{\mathbf{r}}^{R}=\left.\langle\mathbf{S}\rangle_{\mathbf{r}}^{R D}\right|_{\varphi=\phi}$ where the superscript " $\mathrm{R}$ " denotes, similar to the previous ones, that only the Rashba term is nonvanishing.

As an example illustrating the Rashba spin precession (RSP), let $\phi_{s}$ be $\pi / 8$. The spin orientations along nine straight paths are shown in Fig. 1(a) where a compact unit, defined as

$$
R_{0} \equiv 2 \pi \hbar^{2} /\left(m^{*} \sqrt{\alpha^{2}+\beta^{2}}\right),
$$

is used. Note that $R_{0}$ is essentially the precession period length, within which the spin completes one period of precession on the $x$ or $y$ axes, and is typically of the order of or less than $1 \mu \mathrm{m}$ for the Rashba case. ${ }^{17}$ Each pair of adjacent paths includes an angle of $\pi / 8$, which divides a halfcircumferential angle into eight equal parts. Spin precessions are clearly observed, except for the path which is perpendicular to the injected spin [see the $-3 \pi / 8$ path in Fig. 1(a)]. This is reasonably expected since the projection of the injected spin on one of the two eigenspin states, which are always perpendicular to the electron wave vector, vanishes. Thus the fact that only one component of the basis is occupied leads to zero spin precession.

Interestingly, the RSP behaves simply like a windshield wiper swinging about the direction perpendicular to the elec- 
tron wave vector (or the propagation path). Also, it can be observed that the projection of $\langle\mathbf{S}\rangle_{\mathbf{r}}^{R}$ on the direction perpendicular to the path is universally conserved. Physically, these can be understood as direct consequences of the spin precessing about the circular effective magnetic field generated by the Rashba Hamiltonian, leading to such a projection of the spin on the $x-y$ plane. Mathematically, the conservation of the perpendicular-spin projection can also be demonstrated by calculating $\langle\mathbf{S}\rangle_{\mathbf{r}}^{R} \cdot \hat{r}_{\|}$and $\langle\mathbf{S}\rangle_{\mathbf{r}}^{R} \cdot \hat{r}_{\perp}$ where $\hat{r}_{\|}$and $\hat{r}_{\perp}$ are the unit vectors in the directions parallel and perpendicular to the path, respectively. Let us define these two projection quantities to be $\langle\mathbf{S}\rangle_{\mathbf{r}, \|}^{R}$ and $\langle\mathbf{S}\rangle_{\mathbf{r}, \perp}^{R}$. Straightforward mathematics yields

$$
\langle\mathbf{S}\rangle_{\mathbf{r}, \|}^{R}=S_{0, \|} \cos \Delta \theta, \quad\langle\mathbf{S}\rangle_{\mathbf{r}, \perp}^{R}=S_{0, \perp}
$$

where $S_{0, \|}$ and $S_{0, \perp}$ are the projections of the injected spin (with normalized magnitude) on $\hat{r}_{\|}$and $\hat{r}_{\perp}$, respectively.

Furthermore, the transport directions, along which no spin precession occurs, can be mathematically tested by calculating the scalar product of $\langle\mathbf{S}\rangle_{\mathbf{r}}^{R}$ and $\mathbf{S}_{0}$. The result is $\langle\mathbf{S}\rangle_{\mathbf{r}}^{R} \cdot \mathbf{S}_{0}$ $=\cos ^{2}(\Delta \theta / 2)-\cos \left[2\left(\phi-\phi_{s}\right)\right] \sin ^{2}(\Delta \theta / 2)$, which reaches its maximum when $\phi=\phi_{s}+(n+1 / 2) \pi$, with $n$ being an integer. That is, in the directions perpendicular to the injected spin, we always have $\langle\mathbf{S}\rangle_{\mathbf{r}}^{R}=\mathbf{S}_{0}$ [see the $-3 \pi / 8$ path in Fig. 1(a)].

In the absence of the Rashba term, we have $\gamma(\alpha=0)=\beta$ and $\varphi=\pi / 2-\phi$ from Eq. (3). Thus the expectation value obtained in Eq. (5) is modified as

$$
\langle\mathbf{S}\rangle_{\mathbf{r}}^{D}=\left(\begin{array}{c}
\cos \phi_{s} \cos ^{2} \frac{\Delta \theta}{2}+\cos \left(2 \phi+\phi_{s}\right) \sin ^{2} \frac{\Delta \theta}{2} \\
\sin \phi_{s} \cos ^{2} \frac{\Delta \theta}{2}-\sin \left(2 \phi+\phi_{s}\right) \sin ^{2} \frac{\Delta \theta}{2}
\end{array}\right),
$$

where the superscript " $\mathrm{D}$ " is, again, a reminder that only the Dresselhaus term is present. The spin orientations on nine straight paths are plotted in Fig. 1(b). Although the Dresselhaus spin precession (DSP) appears to be more complicated than the Rashba case, it is still analyzable mathematically. We first turn to the projections of $\langle\mathbf{S}\rangle_{\mathbf{r}}^{D}$ on $\hat{r}_{\|}$and $\hat{r}_{\perp}$. Using Eq. (8), we obtain

$$
\begin{aligned}
\langle\mathbf{S}\rangle_{\mathbf{r}, \|}^{D}= & S_{0, \|}\left(\cos ^{2} \frac{\Delta \theta}{2}+\cos 4 \phi \sin ^{2} \frac{\Delta \theta}{2}\right) \\
& -S_{0, \perp} \sin 4 \phi \sin ^{2} \frac{\Delta \theta}{2},
\end{aligned}
$$

and

$$
\begin{aligned}
\langle\mathbf{S}\rangle_{\mathbf{r}, \perp}^{D}= & S_{0, \perp}\left(\cos ^{2} \frac{\Delta \theta}{2}-\cos 4 \phi \sin ^{2} \frac{\Delta \theta}{2}\right) \\
& -S_{0, \|} \sin 4 \phi \sin ^{2} \frac{\Delta \theta}{2} .
\end{aligned}
$$

The projections shown above do not in general exhibit conserved quantities as in the Rashba case, except on two sets of paths: (i) For $\phi=n \pi / 4$, with $n$ being an odd integer, we have $\langle\mathbf{S}\rangle_{\mathbf{r}, \|}^{D}=S_{0, \|} \cos \Delta \theta$ and $\langle\mathbf{S}\rangle_{\mathbf{r}, \perp}^{D}=S_{0, \perp}$, which are exactly the same as Eqs. (7). Thus in these directions, namely, [110], $[\overline{1} 10],[\overline{1} \overline{1} 0]$, and $[1 \overline{1} 0]$ (for which only $[110]$ and $[1 \overline{1} 0]$ are shown in our case), the spin precession behaves like the RSP [see Fig. 1(b)]; (ii) For $\phi=n \pi / 4$ with $n$ being an even integer, we have $\langle\mathbf{S}\rangle_{\mathbf{r}, \|}^{D}=S_{0, \|}$ and $\langle\mathbf{S}\rangle_{\mathbf{r}, \perp}^{D}=S_{0, \perp} \cos \Delta \theta$, which are symmetric to the Rashba case such that the projection of $\langle\mathbf{S}\rangle_{\mathbf{r}}^{D}$ on $\hat{r}_{\|}$is conserved, while that on $\hat{r}_{\perp}$ is oscillatory, leading to another type of "swinging" spin precession on those directions, namely, [100], [010], [100] (which is not shown

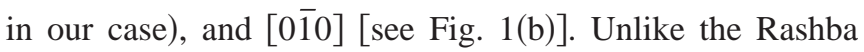
case, in which the eigenspin states are symmetric under rotations of about [001] and the spin precession depends only on the injected spin, these eight directions found above show that there is an intrinsic dependence of the DSP on crystallographic directions.

Similar to the Rashba case, there also exists one straight path on which no spin precession occurs. This can be found, again, by calculating the scalar product of $\langle\mathbf{S}\rangle_{\mathbf{r}}^{D}$ and $\mathbf{S}_{0}$. Using Eq. (8) we obtain $\langle\mathbf{S}\rangle_{\mathbf{r}}^{D} \cdot \mathbf{S}_{0}=\cos ^{2}(\Delta \theta / 2)+\cos [2(\phi$ $\left.\left.+\phi_{0}\right)\right] \sin ^{2}(\Delta \theta / 2)$ showing that for $\phi=-\phi_{0}+n \pi$ the spin precession vanishes [see the $-\pi / 8$ path in Fig. 1(b)].

In the presence of both terms with the same order of coupling strength, the spatial behavior of the spin precession is correspondingly envisioned as the superposition of the RSP and the DSP. Since the DSP behaves like the RSP on the [110], [ $\overline{1} 10],[\overline{1} \overline{1} 0]$, and [1 $1 \overline{1} 0]$ axes, as we have explained previously, the RSP behavior of the electron spin is unavoidably preserved. As an example, the spin precession in the presence of both the Rashba and the Dresselhaus terms is shown in Fig. 2(a), where the coupling ratio is set to be $\alpha / \beta=2.15$, referring to the very recent experimental research on $\alpha / \beta$ in $n$-type InAs QWs. ${ }^{21}$ As expected, the spin preces- (a)

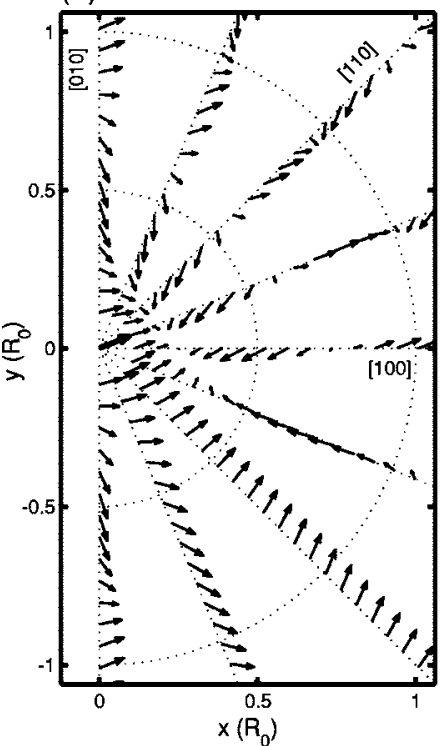

(b)

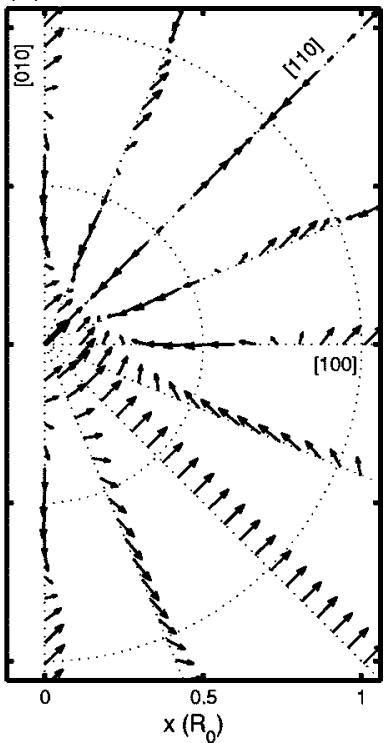

FIG. 2. Spin precession due to Rashba and Dresselhaus spinorbit couplings with the coupling ratio $\alpha / \beta=2.15$ in a 2DEG. Each arrow indicates $\langle\mathbf{S}\rangle_{\mathbf{r}}^{R D}$ on the corresponding space point. The injected spin, shown by the bold arrows on $(0,0)$, is set to orient (a) $\pi / 8$ and (b) $\pi / 4$ with respect to [100]. The compact unit $R_{0}$ is defined in Eq. (6) and the dotted lines are guides for the eyes. 
sion on [110] and [1 $\overline{10}$ ] is still the same as the RSP, while that on other paths seems less expectable. Other QWs, with Rashba and Dresselhaus SO couplings being of the same order (such as InGaAs QWs with a coupling ratio $\alpha / \beta$ ranging between 1.5 and 1.85), ${ }^{21}$ have spin-precession behaviors similar to what we have shown in Fig. 2(a). When either Rashba or Dresselhaus term dominates, the spin precession returns to those shown in Figs. 1(a) or 1(b). A special case of $|\alpha|=|\beta|$, which leads to the cancellation of the $k$ dependence of the eigenstates, ${ }^{25}$ exhibits universal direction $( \pm \pi / 4$ for $\alpha=\mp \beta$ ) about which the electron spin precesses.

Another interesting point is that we can always suppress the spin precession by properly arranging the injected spin. In general, one can first determine $\alpha / \beta$ and then choose the proper orientation of the injected spin corresponding to a certain propagation direction. However, what we emphasize is that on the four RSP-preserved axes, the eigenspinors are always perpendicular to $\mathbf{k}_{\|}$, so that the spin transport is precessionless on these four directions, no matter what the ratio $\alpha / \beta$ is. As an example, when setting the injected spin pointing to $\pi / 4$ we observed a precessionless axis lying on [1 $\overline{1} 0]$ [see Fig. 2(b)]. This supression of spin precession is also consistent with the previous work of Kiselev and Kim. ${ }^{26}$
In conclusion, we have presented the analytical results of the space-dependent expectation values of the spin operator with respect to the injected spin-polarized electron state in the presence of both the Rashba and the Dresselhaus SO couplings. Using the analytical results, the spatial behaviors of the RSP, the DSP, and the composite case are analyzed. The RSP is shown to behave like a swinging wiper, physically stemming from the projection onto the 2DEG layer of the spin precessing around the in-plane effective magnetic field generated by $H_{R}$ and mathematically resulting from the conservation of $\langle\mathbf{S}\rangle_{\mathbf{r}, \perp}^{R}$ and the oscillation of $\langle\mathbf{S}\rangle_{\mathbf{r}, \| y}^{R}$, as shown in Eq. (7). The DSP has a more complicated way of precession, but it is shown to preserve the RSP behavior on the $[110],[\overline{1} 10],[\overline{1} \overline{1} 0]$, and $[1 \overline{1} 0]$ axes. This implies that the spatial behaviors of the spin precession, due to inversion asymmetry in the 2DEG along these four directions, are always invariant and the same as those of the RSP regardless of the influence of the Dresselhaus term. One can therefore envision that a proper choice of the channel direction such as the [110] axis shown in Fig. 2(b)\} in the structure of the Datta-Das transistor may suppress the undesired influence of the Dresselhaus term.

This work was supported by the Republic of China National Science Council Grant No. 93-2112-M-002-011.
${ }^{1}$ Semiconductor Spintronics and Quantum Computation, edited by D. D. Awschalom, D. Loss, and N. Samarth (Springer, Berlin, 2002).

${ }^{2}$ E. I. Rashba, Sov. Phys. Solid State 2, 1109 (1960); Yu. A. Bychkov and E. I. Rashba, JETP Lett. 39, 78 (1984).

${ }^{3}$ B. Das, D. C. Miller, S. Datta, R. Reifenberger, W. P. Hong, P. K. Bhattacharya, J. Singh, and M. Jaffe, Phys. Rev. B 39, 1411 (1989).

${ }^{4}$ J. Luo, H. Munekata, F. F. Fang, and P. J. Stiles, Phys. Rev. B 41, 7685 (1990).

${ }^{5}$ J. Nitta, T. Akazaki, H. Takayanagi, and T. Enoki, Phys. Rev. Lett. 78, 1335 (1997).

${ }^{6}$ J. P. Heida, B. J. van Wees, J. J. Kuipers, T. M. Klapwijk, and G. Borghs, Phys. Rev. B 57, 11911 (1998).

${ }^{7}$ C.-M. Hu, J. Nitta, T. Akazaki, H. Takayanagi, J. Osaka, P. Pfeffer, and W. Zawadzki, Phys. Rev. B 60, 7736 (1999).

${ }^{8}$ D. Grundler, Phys. Rev. Lett. 84, 6074 (2000).

${ }^{9}$ G. Engels, J. Lange, Th. Schäpers, and H. Lüth, Phys. Rev. B 55, R1958 (1997).

${ }^{10}$ G. Dresselhaus, Phys. Rev. 100, 580 (1955).

${ }^{11}$ G. Lommer, F. Malcher, and U. Rössler, Phys. Rev. B 32, 6965 (1985); Yu. A. Bychkov and E. I. Rashba, Proceedings of the 17th International Conference on Physics of Semiconductors, San Francisco, 1984, edited by James D. Chadi and Walter A. Harrison (Springer, New York, 1985), p. 321; M. I. D’yakonov and V. Y. Kachorovskii, Sov. Phys. Semicond. 20, 110 (1986).

${ }^{12}$ M. I. Dyakonov and V. I. Perel, Sov. Phys. JETP 33, 1053 (1971).

${ }^{13}$ G. Bastard and R. Ferreira, Surf. Sci. 267, 335 (1992).

${ }^{14}$ L. Vervoort and P. Voisin, Phys. Rev. B 56, R12 744 (1997).
${ }^{15}$ U. Rössler and J. Kainz, Solid State Commun. 121, 313 (2002).

${ }^{16}$ G. Lommer, F. Malcher, and U. Rossler, Phys. Rev. Lett. 60, 728 (1988).

${ }^{17}$ S. Datta and B. Das, Appl. Phys. Lett. 56, 665 (1990).

${ }^{18}$ R. Winkler, Phys. Rev. B 69, 045317 (2004).

${ }^{19}$ A. Lusakowski, J. Wróbel, and T. Dietl, Phys. Rev. B 68, R081201 (2003).

${ }^{20}$ B. Jusserand, D. Richards, G. Allan, C. Priester, and B. Etienne, Phys. Rev. B 51, 4707 (1995); W. Knap, C. Skierbiszewski, A. Zduniak, E. Litwin-Staszewska, D. Bertho, F. Kobbi, J. L. Robert, G. E. Pikus, F. G. Pikus, S. V. Iordanskii, V. Mosser, K. Zekentes, and Yu. B. Lyanda-Geller, ibid. 53, 3912 (1996); J. B. Miller, D. M. Zumbühl, C. M. Marcus, Y. B. Lyanda-Geller, D. Goldhaber-Gordon, K. Campman, and A. C. Gossard, Phys. Rev. Lett. 90, 076807 (2003).

${ }^{21}$ S. D. Ganichev, V. V. Bel'kov, L. E. Golub, E. L. Ivchenko, P. Schneider, S. Giglberger, J. Eroms, J. De Boeck, G. Borghs, W. Wegscheider, D. Weiss, and W. Prettl, Phys. Rev. Lett. 92, 256601 (2004).

${ }^{22}$ David Z.-Y. Ting and Xavier Cartoixà, Phys. Rev. B 68, 235320 (2003).

${ }^{23}$ John Schliemann and Daniel Loss, Phys. Rev. B 68, 165311 (2003).

${ }^{24}$ J. J. Sakurai, Modern Quantum Mechanics, Revised Edition, (Addison-Welsey, Reading, MA, 1994).

${ }^{25}$ John Schliemann, J. Carlos Egues, and Daniel Loss, Phys. Rev. Lett. 90, 146801 (2003).

${ }^{26}$ A. A. Kiselev and K. W. Kim, Phys. Rev. B 61, 13115 (2000). 